\title{
Papers
}

\section{Influence of psychological coping on survival and recurrence in people with cancer: systematic review}

Mark Petticrew, Ruth Bell, Duncan Hunter

\begin{abstract}
Objective To summarise the evidence on the effect of psychological coping styles (including fighting spirit, helplessness/hopelessness, denial, and avoidance) on survival and recurrence in patients with cancer. Design Systematic review of published and unpublished prospective observational studies. Main outcome measures Survival from or recurrence of cancer.

Results 26 studies investigated the association between psychological coping styles and survival from cancer, and 11 studies investigated recurrence. Most of the studies that investigated fighting spirit (10 studies) or helplessness/hopelessness (12 studies) found no significant associations with survival or recurrence. The evidence that other coping styles play an important part was also weak. Positive findings tended to be confined to small or methodologically flawed studies; lack of adjustment for potential confounding variables was common. Positive conclusions seemed to be more commonly reported by smaller studies, indicating potential publication bias.

Conclusion There is little consistent evidence that psychological coping styles play an important part in survival from or recurrence of cancer. People with cancer should not feel pressured into adopting particular coping styles to improve survival or reduce the risk of recurrence.
\end{abstract}

\section{Introduction}

It is a popular belief that psychological factors can influence survival from cancer, particularly breast cancer. ${ }^{1}$ Current research interest in this subject stems from 1979 when a small UK study found that a psychological coping style characterised by a "fighting spirit" was associated with longer survival from breast cancer. A more negative style of coping characterised as "helplessness/hopelessness" has also been reported to predict a poorer outcome, though not all studies have found such an association. ${ }^{2-6}$ It is important to know whether these psychological factors do have an influence on survival because psychological interventions have been developed to enhance the use of certain coping styles to prolong survival, and there is strong lay and professional support for such therapies. ${ }^{7}$
Such as association is biologically plausible, and several possible mechanisms have been proposed-for example, through immunological and neuroendocrine mechanisms. ${ }^{28}$ However there are conflicting views regarding the importance of coping styles in the progression of cancer, ranging from the view that they have an important influence to the view that the theory is characterised by myth and anecdote. ${ }^{9} 10$

We carried out a comprehensive systematic review to assess the strength of the evidence for an association between psychological coping and cancer outcome.

\section{Methods}

Search strategy-Following systematic review guidelines $^{11}{ }^{12}$ we searched several databases for published and unpublished studies (in any language) on the association between progression of cancer, recurrence or survival, and psychological coping: Medline 1966-June 2002, PsycINFO 1887-June 2002, ASSIA 1987-June 2002, Embase 1980-June 2002, Cancerlit 1966-June 2002, Dissertation Abstracts 1975-June 2002, the NLM gateway (accessed 21 June 2002), and CINAHL 1982-June 2002. We searched bibliographies and reviews and contacted key individuals and authors for additional unpublished information when necessary.

Inclusion and exclusion criteria-We included prospective cohort studies that included mortality, survival, or recurrence as outcomes. We excluded studies of the association between coping and immune responses or other biochemical markers, if this was the only outcome reported, and studies of personality types (for example, "type C" personality).

Data extraction and validity assessment-When the results of both multivariate analyses and univariate analyses were presented we extracted data from the multivariate analysis and noted the variables used in the adjustment (table 1 and 2). When necessary we contacted authors for unpublished data; one author supplied the requested information. Data were extracted by one reviewer and checked by a second. The studies were assessed independently by two reviewers against three methodological criteria: whether the sample represented an inception cohort, the degree of adjustment for potential confounders, and whether the assessment of coping was carried out early in the disease process. The results were summarised narratively.
MRC Social and
Public Health
Sciences Unit,
Glasgow G12 8RZ
Mark Petticrew
associate director
Department of
Epidemiology and
Public Health,
Medical School,
University of
Newcastle,
Newcastle upon
Tyne NE2 4HH
Ruth Bell
lecturer
Community Health
and Epidemiology,
Abramsky Hall,
Queens University
Kingston, Ontario,
Canada K7L 3N6
Duncan Hunter
assistant professor
Correspondence to:
M Petticrew
mark@msoc.mrc.
gla.ac.uk
bmj.com 2002;325:1066 


\section{Results}

We found 26 studies that investigated the association between psychological coping and survival and 11 studies that investigated recurrence (figure). Some studies were reported in more than one paper-for example, results pertaining to different follow up periods. The most common diagnosis of patients in these studies was breast cancer, though we also found studies that investigated leukaemia, melanoma, and lung and gastrointestinal cancers, with follow up periods ranging from several months to 15 years (tables 1, 2, and 3).

\section{Assessment of validity}

Thirteen studies met all three methodological criteria. Table 3 shows methodological details of each study. Table 1 shows studies of survival, and table 2 shows studies of recurrence. About a third of all studies did not adjust for potential confounding variables. Most of the studies were small; the overall median sample size was 125 , and only four studies recruited more than 200 patients. There was no association between study quality (scored 1 to 3 , see tables 1 and 2) and study outcome (presence versus absence of significant findings; $\chi^{2}$ test for trend; $\mathrm{P}=0.5$ ). Where studies are referred to as "small" this is defined as "smaller than the median study size."

\section{Findings}

Fighting spirit-Ten studies investigated the impact of "fighting spirit" on survival. ${ }^{2}{ }^{5-7}$ 13-20 Positive findings that linked use of this coping style to longer survival were confined to two small studies (table 1). ${ }^{2-5}{ }^{20}$ Four small studies examined the association with recurrence of cancer. Three studies reported that fighting spirit was associated with a reduced risk..$^{2-46}{ }^{15}$ This finding was not confirmed by the fourth, larger study $(n=578){ }^{7}$

Helplessness/hopelessness-Twelve studies examined hopelessness/helplessness as a predictor of reduced survival in cancer patients. ${ }^{2-4} 67$ 13-19 21-25 Only two small studies reported that more frequent such feelings adversely affected survival. ${ }^{23}$ Five studies presented

\begin{tabular}{|c|}
\hline $\begin{array}{l}\text { Potentially relevant studies of psychological states and } \\
\text { cancer identified and screened for retrieval }(n=664)\end{array}$ \\
\hline$\downarrow$ \\
\hline $\begin{array}{l}\text { Excluded: not primary studies of psychological } \\
\text { states and cancer in humans }(n=269)\end{array}$ \\
\hline$\downarrow$ \\
\hline Papers retrieved for further evaluation $(n=395)$ \\
\hline$\downarrow$ \\
\hline $\begin{array}{l}\text { Excluded from review ( } n=361) \\
\text { - Not studies of cancer }(n=126) \\
\text { - Studies of stress, but not coping ( } 20 \text { case control studies of } \\
\text { psychological stress and cancer risk; } 20 \text { other studies of stress) } \\
\text { - Other studies of cancer and life events ( } n=24) \\
\text { - Reviews or commentary papers }(n=120) \\
\text { - Studies of personality types or psychological or social factors, and } \\
\text { cancer risk or outcome }(n=51)\end{array}$ \\
\hline$\downarrow$ \\
\hline Papers included with usable information $(n=37)$ \\
\hline
\end{tabular}

Flowchart for main search. Search terms included: (cancer\$ or neoplasm\$), expanded in Medline and other databases where possible and denial or coping or attitude or fighting spirit or avoidance or hope\$ and (prognos\$ or relapse or recurrence or survival or progression) data on recurrence of cancer, but the findings were inconsistent. ${ }^{6} 752122{ }^{26}$ In one study, few data were presented $^{15}$ and in another the outcome variable was a composite variable based on a 13 point indicator of clinical status. ${ }^{26}$ The two other studies that reported associations with recurrence were small or limited by methodological problems, or both. In particular, there was limited control of confounding. ${ }^{2122}$ The recent large UK study $(\mathrm{n}=578)$, while of higher quality, reported mixed findings: helplessness/hopelessness predicted recurrence when those with high and low scores were compared but not when it was the predominant coping style.

Denial or avoidance-Denial or avoidance were assessed in 15 studies of survival; 10 of these investigated avoidance ${ }^{178131417-1927-29}$ and five investigated denial. ${ }^{2-4} 6153031$ These studies did not report any significant independent associations between the use of an avoidant style of coping and survival. There was also little evidence to suggest that denial was an important predictor of survival ${ }^{17132728}$ : two studies reported an association between denial and survival but one presented no supporting data. ${ }^{30}$ The other small study found that the use of denial predicted death from breast cancer at 10 and 15 years. ${ }^{2-4}$ Eight studies explored the effects of denial or avoidance on recurrence of cancer. $^{2-4}$ 6-8 15203233 Only one of these studies (a small study carried out in patients with breast cancer) reported that denial predicted recurrence. ${ }^{2-4}$ This association was not reported in other larger studies. ${ }^{78}$

Stoic acceptance and fatalism-Nine studies explored the impact of acceptance and fatalism, ${ }^{2}$ ( $^{13-19}$ and none of the four higher quality studies found that they predicted survival. ${ }^{731516}$ The evidence regarding recurrence of cancer was similarly weak..$^{26} 715$ The only study that reported a significant association presented no supporting data. ${ }^{15}$

Anxious coping/anxious preoccupation, depressive coping-Ten studies investigated the impact of an anxious or depressive coping style on survival. ${ }^{6} 7^{14-19}$ 34-40 One small study reported that higher anxious preoccupation scores predicted shorter survival, ${ }^{13}$ and a study of 103 patients found that the use of depressive coping predicted shorter survival. ${ }^{39}{ }^{40}$ Three studies presented relative risks associated with anxious preoccupation, all of which were close to $1.0 .^{71318}{ }^{19}$ One small study (n=35) reported an association between depression and survival, though this study had methodological drawbacks with respect to patient recruitment and confounding. ${ }^{38}$ None of these psychological factors was reported to be significantly associated with recurrence of cancer.

Active or problem focused coping-Eight studies explored the effects of active or problem focused cop-

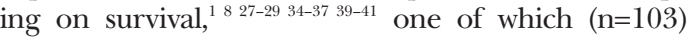
reported that the use of active coping was a predictor of longer survival up to seven years. ${ }^{39} 40$ The largest study ( $\mathrm{n}=847$ ) compared high, medium, and low users of this coping style and found no association with survival after they controlled for clinical and sociodemographic factors. ${ }^{1}$ Another study (n=133), which investigated a coping style labelled "coping by control," reported no significant findings. ${ }^{41}$ Active or problem focused coping was not associated with recurrence.

Emotional factors (including suppression of emotions and emotion focused coping)-We identified six studies on survival. ${ }^{1} 7_{23} 293^{34-37}$ One study ( $\left.\mathrm{n}=847\right)$ met the three 
Table 1 Prospective studies of survival from cancer and coping style* (posted as supplied by authors)

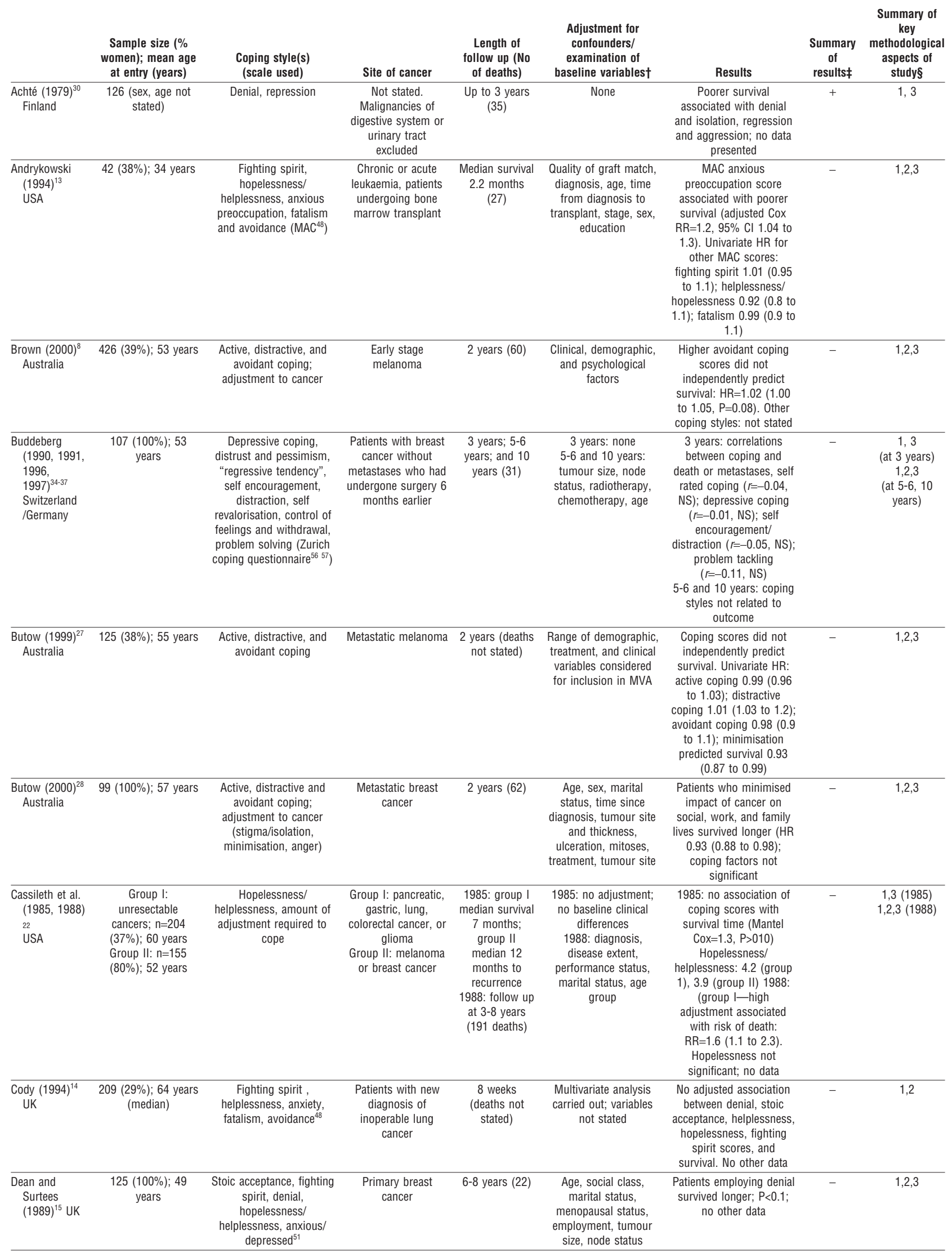


Table 1 Prospective studies of survival from cancer and coping style* (posted as supplied by authors) contd

\begin{tabular}{|c|c|c|c|c|c|c|c|c|}
\hline & $\begin{array}{l}\text { Sample size (\% } \\
\text { women); mean age } \\
\text { at entry (years) }\end{array}$ & $\begin{array}{l}\text { Coping style(s) } \\
\text { (scale used) }\end{array}$ & Site of cancer & $\begin{array}{l}\text { Length of } \\
\text { follow up (No } \\
\text { of deaths) }\end{array}$ & $\begin{array}{c}\text { Adjustment for } \\
\text { confounders/ } \\
\text { examination of } \\
\text { baseline variables } †\end{array}$ & Results & $\begin{array}{c}\text { Summary } \\
\text { of } \\
\text { resultsł }\end{array}$ & $\begin{array}{c}\text { Summary of } \\
\text { key } \\
\text { methodologica } \\
\text { aspects of } \\
\text { study§ }\end{array}$ \\
\hline $\begin{array}{l}\text { Derogatis } \\
(1979)^{38} \\
\text { USA }\end{array}$ & $35(100 \%) ; 55$ years & $\begin{array}{l}\text { Anxiety, depression, } \\
\text { hostility, guilt, } \\
\text { psychoticism, } \\
\text { psychological symptom } \\
\text { severity (defined by } \\
\text { authors as coping styles) }\end{array}$ & $\begin{array}{l}\text { Metastatic breast } \\
\text { cancer }\end{array}$ & $\begin{array}{l}\text { Long term } \\
\text { survivors } \\
\text { (mean=23 } \\
\text { months) } v \\
\text { short term } \\
\text { survivors } \\
\text { (mean=9 } \\
\text { months) (13) }\end{array}$ & $\begin{array}{l}\text { None. No baseline } \\
\text { differences in age, } \\
\text { disease-free interval, \% } \\
\text { premenopausal, } \\
\text { metastases, Karnofsky } \\
\text { score, response to } \\
\text { therapy }\end{array}$ & $\begin{array}{c}\text { Survivors } v \\
\text { non-survivors: hostility } \\
(t=2.4, P<0.01), \\
\text { psychoticism }(t=2.7, \\
P<0.01), \text { depression } \\
(t=2.3, P<0.05), \text { guilt } \\
(t=2.6, P<0.05), \text { negative } \\
\text { affect }(t=2.7, P<0.01) \text {, } \\
\text { affect balance }(t=2.1 \text {, } \\
P<0.05)\end{array}$ & + & 3 \\
\hline $\begin{array}{l}\text { Faller (1997; } \\
\text { 1999; and } \\
\text { unpublished } \\
\text { data) } \\
\text { Germany }\end{array}$ & $103(17 \%) ; 59$ years & $\begin{array}{l}\text { Depressive coping, active } \\
\text { coping, } \text { hope }^{35} 57\end{array}$ & $\begin{array}{c}\text { Lung cancer } \\
\text { Stage 1: } 2 \%, \\
\text { Stage II: } 7 \% \\
\text { Stage IIla/IIIb: } 49 \% \\
\text { Stage IV: } 42 \%\end{array}$ & $\begin{array}{l}\text { (i) } 3-5 \text { years } \\
\text { (ii) } 5-7 \text { years } \\
\text { (92) }\end{array}$ & $\begin{array}{l}\text { (i) Karnofsky status, } \\
\text { treatment, stage } \\
\text { (ii) Histology, stage, } \\
\text { Karnofsky status, } \\
\text { treatment }\end{array}$ & $\begin{array}{c}\text { Use of active coping } \\
\text { predicted longer survival } \\
\text { (3-5 years RR=1.91, } 1.17 \\
\text { to } 3.12 \text {; also } 5-7 \text { years } \\
\text { RR=0.68, } 0.50 \text { to } 0.94 \text { ). } \\
\text { Use of depressive coping } \\
\text { predicted shorter survival } \\
\text { (RR=1.88, } 1.29 \text { to } 2.73 \text { ) }\end{array}$ & 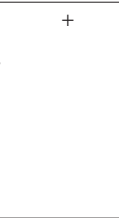 & $1,2,3$ \\
\hline $\begin{array}{l}\text { Giraldi }(1997)^{16} \\
\text { Italy }\end{array}$ & $95(100 \%) ; 51$ years & $\begin{array}{c}\text { Fighting spirit, } \\
\text { hopelessness, anxious } \\
\text { preoccupation, fatalism }{ }^{48}\end{array}$ & $\begin{array}{l}\text { Breast cancer } \\
\text { T1: } 36 \% \\
\text { T2: } 41 \%\end{array}$ & $\begin{array}{l}6 \text { years } \\
(15)\end{array}$ & $\begin{array}{l}\text { Nodal status, histology, } \\
\text { tumour stage, } \\
\text { oestrogen receptor } \\
\text { status }\end{array}$ & $\begin{array}{c}\text { High } v \text { low fighting spirit } \\
\text { score: } \mathrm{RR}=1.08,0.94 \text { to } \\
1.23 \\
\text { High } v \text { low hopelessness } \\
\text { score: unadjusted } \\
\mathrm{RR}=0.9,0.72 \text { to } 1.12 ; \text { no } \\
\text { other data }\end{array}$ & 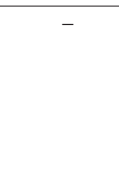 & $1,2,3$ \\
\hline $\begin{array}{l}\text { Greer }(1979),{ }^{2} \\
\text { Pettingale } \\
(1984),{ }^{3} \\
\text { Pettingale } \\
(1985),{ }^{4} \\
\text { Greer } \\
(1990)^{5} \text { UK }\end{array}$ & $\begin{array}{c}69(100 \%) \text {; aged } \\
<70 \text { years }\end{array}$ & $\begin{array}{l}\text { Denial, fighting spirit, } \\
\text { stoic acceptance, } \\
\text { helplessness/ } \\
\text { hopelessness }\end{array}$ & $\begin{array}{c}\text { Breast cancer with } \\
\text { no metastases }\end{array}$ & $\begin{array}{c}5,10,15 \text { years } \\
(34,36,47)\end{array}$ & $\begin{array}{c}5 \text { years: none } \\
10 \text { years: age, } \\
\text { menopausal status, } \\
\text { stage, operation, } \\
\text { radiotherapy, histology } \\
15 \text { years: age, } \\
\text { menopausal status, } \\
\text { stage, operation, } \\
\text { radiotherapy, histology }\end{array}$ & $\begin{array}{c}5 \text { years: use of stoic } \\
\text { acceptance or } \\
\text { helplessness/ } \\
\text { hopelessness and risk of } \\
\text { death } R R=4.4,1.1 \text { to } \\
17.0) \\
10 \text { years: use of denial or } \\
\text { fighting spirit predicted } \\
\text { death, and death from } \\
\text { breast cancer ( } P=0.003) \\
15 \text { years: denial, fighting } \\
\text { spirit predicted death } \\
\text { ( } z=2.83, P=0.006) \text {, death } \\
\text { from cancer }(z=3.6, \\
P<0.001)\end{array}$ & $\begin{array}{l}+ \\
+ \\
+\end{array}$ & $\begin{array}{c}1,3 \text { (at } 5 \text { years) } \\
1,2,3 \text { (at } 10 \\
\text { years) } \\
1,2,3 \text { (at } 10 \\
\text { years) }\end{array}$ \\
\hline $\begin{array}{l}\text { Hislop }(1987)^{41} \\
\text { Canada }\end{array}$ & $\begin{array}{l}133(100 \%) ;<55 \\
\text { years at diagnosis }\end{array}$ & $\begin{array}{l}\text { Coping by change, by } \\
\text { control, by stress }\end{array}$ & $\begin{array}{l}\text { Primary ductal } \\
\text { breast cancer }\end{array}$ & $\begin{array}{l}4 \text { years } \\
(26)\end{array}$ & $\begin{array}{c}\text { Age, stage, pathological } \\
\text { nodal status, } \\
\text { histological grade, ER } \\
\text { status }\end{array}$ & $\begin{array}{l}\text { Coping by change (more } \\
v \text { less often): } \text { Cox } \\
\text { adjusted } R R=0.59 \text {, no } \mathrm{Cl} \text {, } \\
\mathrm{P}=0.3 \text { Coping by control: } \\
\text { RR }=0.59 \text { Coping by } \\
\text { stress: } \mathrm{RR}=1.4, \mathrm{P}=0.6\end{array}$ & - & $1,2,3$ \\
\hline $\begin{array}{l}\text { Molassiotis } \\
\quad(1997)^{23} \text { UK }\end{array}$ & $31(29 \%) ; 34$ years & $\begin{array}{l}\text { Emotion focused coping, } \\
\text { humour, withdrawal, } \\
\text { acceptance, hopefulness } \\
\text { (Jalowiec coping scale }^{52} \text { ) }\end{array}$ & $\begin{array}{l}\text { Patients with bone } \\
\text { marrow transplant: } \\
\text { haematological } \\
\text { malignancies }\end{array}$ & $1-2$ years $(20)$ & $\begin{array}{l}\text { Age, sex, marital } \\
\text { status, ethnicity, } \\
\text { diagnosis, quality of } \\
\text { raft match, stage, } \\
\text { previous treatment }\end{array}$ & $\begin{array}{c}\text { Frequent use of hope } \\
\text { (Cox adjusted } R R=0.11 \text {, } \\
0.02 \text { to } 0.52) \text { and less } \\
\text { frequent use of } \\
\text { acceptance }(1.4,1.3 \text { to } \\
\text { 13.4) predicted long term } \\
\text { survival }\end{array}$ & s. & $1,2,3$ \\
\hline $\begin{array}{l}\text { Morris }(1992)^{6} \\
\text { UK }\end{array}$ & $\begin{array}{c}107(100 \%) \\
61(26 \%)\end{array}$ & $\begin{array}{c}\text { Denial, fighting spirit, } \\
\text { anxious preoccupation, } \\
\text { stoic acceptance, } \\
\text { helpless/hopelessness }\end{array}$ & $\begin{array}{c}\text { Breast cancer } \\
(\mathrm{n}=107) \\
\text { Lymphoma }(\mathrm{n}=61)\end{array}$ & $\begin{array}{l}\text { Up to } 5 \text { years } \\
(20)\end{array}$ & $\begin{array}{l}\text { Age, sex, stage, } \\
\text { tumour grade, } \\
\text { non-Hodgkin's } \\
\text { lymphoma, type of } \\
\text { operation, tumour size, } \\
\text { positive nodal status }\end{array}$ & $\begin{array}{l}\text { Breast cancer: no coping } \\
\text { style predicted survival } \\
\text { Lymphoma: anxious } \\
\text { preoccupation/stoicism/ } \\
\text { helplessness-hopelessness } \\
\text { associated with survival. } \\
\text { All cases: association } \\
\text { found between } \\
\text { "prognostic index" and } \\
\text { primary coping style }\left(\chi^{2}\right. \\
\text { for survival } 5.49, P=0.02)\end{array}$ & $\begin{array}{c}- \text { (breast } \\
\text { cancer) } \\
+ \\
\text { (lymphoma) } \\
+ \text { (all } \\
\text { cases) } \\
\end{array}$ & 1,2 \\
\hline $\begin{array}{l}\text { Murphy } \\
(1996)^{17} \text { UK }\end{array}$ & $56(48 \%) ; 35$ years & $\begin{array}{c}\text { Fighting spirit, } \\
\text { helplessness, anxiety, } \\
\text { fatalism, avoidance }{ }^{48}\end{array}$ & $\begin{array}{l}\text { Patients with bone } \\
\text { marrow transplant: } \\
\text { leukaemia: } 68 \% \\
\text { lymphoma: } 25 \% \\
\text { aplastic anaemia: } \\
8 \%\end{array}$ & $\begin{array}{l}\text { Mean follow } \\
\text { up: } 82 \text { months } \\
(18)\end{array}$ & Unadjusted & $\begin{array}{l}\text { No difference in mean } \\
\text { survival time between } \\
\text { patients with fighting } \\
\text { spirit as predominant } \\
\text { coping style and others } \\
\text { ( } P=0.79 \text {; no other data). }\end{array}$ & - & 1,3 \\
\hline
\end{tabular}


Table 1 Prospective studies of survival from cancer and coping style* (posted as supplied by authors) contd

\begin{tabular}{|c|c|c|c|c|c|c|c|c|}
\hline & $\begin{array}{l}\text { Sample size (\% } \\
\text { women); mean age } \\
\text { at entry (years) }\end{array}$ & $\begin{array}{l}\text { Coping style(s) } \\
\text { (scale used) }\end{array}$ & Site of cancer & $\begin{array}{l}\text { Length of } \\
\text { follow up (No } \\
\text { of deaths) }\end{array}$ & $\begin{array}{c}\text { Adjustment for } \\
\text { confounders/ } \\
\text { examination of } \\
\text { baseline variablest }\end{array}$ & Results & $\begin{array}{l}\text { Summary } \\
\text { of } \\
\text { results }\end{array}$ & $\begin{array}{c}\text { Summary of } \\
\text { key } \\
\text { methodological } \\
\text { aspects of } \\
\text { study§ }\end{array}$ \\
\hline $\begin{array}{l}\text { Nordin and } \\
\text { Glimelius } \\
(1997, \\
\text { 1998) } \\
\text { Sweden }\end{array}$ & $139(51 \%) ; 67$ years & $\begin{array}{c}\text { Fighting spirit, } \\
\text { helplessness, anxious } \\
\text { preoccupation, anxiety, } \\
\text { fatalism, avoidance }{ }^{48}\end{array}$ & $\begin{array}{c}\text { Gastrointestinal } \\
\text { cancers (colon: } \\
27 \% \text {, rectum: } 23 \% \text {, } \\
\text { gastric: } 24 \% \text {, } \\
\text { pancreatic: } 16 \%, \\
\text { biliary: } 12 \% \text { ) }\end{array}$ & $\begin{array}{c}\leqslant 12 \text { months } \\
(56)\end{array}$ & $\begin{array}{l}\text { No adjustment; cured } \\
\text { and non-cured patients } \\
\text { did not differ in age, } \\
\text { time since diagnosis, } \\
\text { or sex }\end{array}$ & $\begin{array}{c}\text { At } 6 \text { months: fighting } \\
\text { spirit as predominant } \\
\text { style RR=0.86, } 0.61 \text { to } \\
\text { 1.19); helplessness/ } \\
\text { hopelessness } 1.19,0.84 \\
\text { to } 1.69) ; \text { anxious } \\
\text { preoccupation } 1.03 \text { ( } 0.7 \\
\text { to } 1.5) \text {; fatalism } 0.88 \text {, } \\
0.56 \text { to } 1.39 \text { ) }\end{array}$ & - & 1,3 \\
\hline $\begin{array}{l}\text { Reynolds } \\
\qquad(2000)^{1} \text { USA }\end{array}$ & $847(100 \%)$ & $\begin{array}{l}\text { Expressing/suppressing } \\
\text { emotions, wishful } \\
\text { thinking, problem solving, } \\
\text { positive reappraisal, } \\
\text { avoidance, escapism, size } \\
\text { of "coping repertoire" }\end{array}$ & Breast cancer & $\begin{array}{l}\text { Up to } 9 \text { years } \\
\text { after diagnosis } \\
(218)\end{array}$ & $\begin{array}{l}\text { Age, study location, } \\
\text { race, stage, } \\
\text { comorbidity, weight, } \\
\text { progesterone and } \\
\text { oestrogen receptor } \\
\text { status }\end{array}$ & $\begin{array}{c}\text { Expressing emotions } \\
\text { increased survival } \\
\text { (HR=0.6, } 0.4 \text { to } 0.9) ; \\
\text { suppression reduced } \\
\text { survival }(1.4,1.1 \text { to } 1.9) \text {; } \\
\text { wishful thinking } 0.9,0.7 \\
\text { to } 1.3) \text {; problem solving } \\
0.8,0.6 \text { to } 1.2) ; \text { positive } \\
\text { reappraisal } 1.1,0.7 \text { to } \\
\text { 1.6); avoidance } 0.8,0.6 \\
\text { to } 1.1) ; \text { escapism } 1.1,0.7 \\
\text { to } 1.5) \text {. HRs refer to high } \\
v \text { low use of coping style }\end{array}$ & $+1-$ & $1,2,3$ \\
\hline $\begin{array}{l}\text { Richardson } \\
\quad(1990)^{29} \text { USA }\end{array}$ & $\begin{array}{l}139(35 \%) ; \text { from } \\
<20 \text { to } \geqslant 80 \text { years }\end{array}$ & $\begin{array}{l}\text { Behavioural and cognitive } \\
\text { coping, including } \\
\text { avoidance, information } \\
\text { seeking, problem solving, } \\
\text { affective regulation }{ }^{53}\end{array}$ & $\begin{array}{c}\text { Haematological } \\
\text { malignancies }(n=92) \\
\text { Rectal cancer }(n=47)\end{array}$ & $\begin{array}{c}4 \text { years } 10 \\
\text { months (rectal } \\
\text { cancer); } \leqslant 5 \\
\text { years } \\
\text { (haematological } \\
\text { cancers) (64) }\end{array}$ & Unadjusted & $\begin{array}{l}\text { No significant association } \\
\text { with outcome in either } \\
\text { group. RR for high } v \text { low } \\
\text { avoidance: (i) } 1.08,0.32 \\
\text { to } 2.44 \text {; (ii) } 0.71,0.32 \text { to } \\
1.62 \text { ) }\end{array}$ & - & 1,3 \\
\hline $\begin{array}{l}\text { Ringdal } \\
(1995),)^{25} \\
(1996)^{24} \text { USA }\end{array}$ & $253(45 \%) ; 57$ years & $\begin{array}{l}\text { Hopelessness (Beck } \\
\text { hopelessness scale }{ }^{54} \text { ) }\end{array}$ & $\begin{array}{c}\text { Breast: }(25 \%) \\
\text { Gastrointestinal: } \\
(11 \%) \\
\text { Prostate: } 12 \% \\
\text { Lung: } 11 \%) \\
\text { Lymphomas: } 13 \% \\
\end{array}$ & $\begin{array}{l}\text { Mean survival } \\
\text { time } 7 \text { months } \\
\text { (131) }\end{array}$ & $\begin{array}{l}\text { Age, sex, treatment } \\
\text { intention, physical } \\
\text { functioning, cancer } \\
\text { type, treatment } \\
\text { modality, relapse }\end{array}$ & $\begin{array}{l}\text { Cox unadjusted RR for } \\
\text { hopelessness } 1.11,1.06 \\
\text { to } 1.17) \text {. Association } \\
\text { disappeared in } \\
\text { multivariate analysis }\end{array}$ & - & 2,3 \\
\hline $\begin{array}{l}\text { Schulz }(1996)^{55} \\
\text { USA }\end{array}$ & $\begin{array}{c}268(51 \%) ;>30 \\
\text { years }\end{array}$ & Pessimism, optimism & $\begin{array}{c}\text { Recurrent/ } \\
\text { metastasised breast: } \\
23 \% \\
\text { Lung: } 20 \% \\
\text { Head/neck: } 13 \% \\
\text { Gynaecological: } 9 \% \\
\text { Other cancers }\end{array}$ & 8 months $(70)$ & $\begin{array}{c}\text { Type of cancer, } \\
\text { symptoms at baseline }\end{array}$ & $\begin{array}{l}\text { No direct or indirect } \\
\text { effects of optimism score } \\
\text { on mortality. Pessimism } \\
\text { score did not } \\
\text { independently predict } \\
\text { mortality (adjusted } \\
\text { RR=1.1, } 0.97 \text { to } 1.2 \text { ) }\end{array}$ & - & 2,3 \\
\hline $\begin{array}{l}\text { Silberfarb } \\
\qquad(1991)^{31} \text { USA }\end{array}$ & $\begin{array}{c}290(46 \%) ; \\
13 \%<49 \text { years } 27 \% \\
<59 \text { years } 33 \%<69 \\
\text { years }\end{array}$ & Denial of illness & $\begin{array}{l}\text { Previously untreated } \\
\text { patients with } \\
\text { multiple myeloma }\end{array}$ & $\begin{array}{l}2 \text { years (deaths } \\
\text { not stated) }\end{array}$ & $\begin{array}{l}\text { Age, tumour cell load, } \\
\text { creatinine, "usual } \\
\text { prognostic factors" }\end{array}$ & $\begin{array}{l}\text { No association of denial } \\
\text { with survival; no other } \\
\text { data }\end{array}$ & - & 1,3 \\
\hline $\begin{array}{l}\text { Tschuschke } \\
(2001)^{20} \\
\text { Germany }\end{array}$ & $52(31 \%) ; 36$ years & $\begin{array}{c}\text { Passive } \\
\text { reception/resignation, } \\
\text { distraction, cognitive } \\
\text { structuring, social } \\
\text { contact, compliance, } \\
\text { fighting spirit }\end{array}$ & $\begin{array}{l}\text { Patients with acute } \\
(\mathrm{n}=33) \text { and chronic } \\
(\mathrm{n}=19) \text { myeloid } \\
\text { leukaemia } \\
\text { undergoing bone } \\
\text { marrow } \\
\text { transplantation }\end{array}$ & $\begin{array}{l}\text { Mean survival } \\
\text { time } 2.6 \text { years } \\
(21)\end{array}$ & $\begin{array}{l}\text { Stage, leukaemia type, } \\
\text { age, sex. Distraction, } \\
\text { fighting spirit } \\
\text { subgroups similar in } \\
\text { education, marital } \\
\text { status, social support, } \\
\text { GvHD prophylaxis }\end{array}$ & $\begin{array}{c}\text { Distraction (Wald=7.27, } \\
\mathrm{P}=0.007), \text { fighting spirit } \\
(6.31, \mathrm{P}<0.012) \\
\text { associated with survival. } \\
\text { RRs estimated from } \\
\text { survival curve: high } v \text { low } \\
\text { distraction } 0.61 ; \text { high } v \\
\text { low fighting spirit } 0.65\end{array}$ & + & 2,3 \\
\hline $\begin{array}{l}\text { Watson }(1999)^{7} \\
\text { UK }\end{array}$ & $\begin{array}{c}578(100 \%) ; 55 \\
\text { years }\end{array}$ & $\begin{array}{c}\text { Fighting spirit, } \\
\text { helplessness/ } \\
\text { hopelessness, anxious } \\
\text { preoccupation, fatalism, } \\
\text { avoidance (MAC scale }{ }^{48} \text { ), } \\
\text { emotional suppression }\end{array}$ & $\begin{array}{l}\text { Stage I and II breast } \\
\text { cancer }\end{array}$ & 5 years (133) & $\begin{array}{l}\text { Histopathological } \\
\text { grade, number of +ve } \\
\text { lymph nodes, tumour } \\
\text { size, type of operation, } \\
\text { chemotherapy } v \\
\text { endocrine therapy, } \\
\text { oestrogen receptor } \\
\text { status }\end{array}$ & $\begin{array}{c}\text { No effect of predominant } \\
\text { coping style, or emotional } \\
\text { suppression. Fighting } \\
\text { spirit } \mathrm{HR}=0.86,0.42 \text { to } \\
1.76 ; \mathrm{H} / \mathrm{H} 0.95,0.46 \text { to } \\
1.98 \text {; anxiety } \\
\text { preoccupation 1.04, } 0.5 \\
\text { to } 2.2 ; \text { fatalism } 1.02,0.49 \\
\text { to } 2.15 \text {; avoidance } 1.53 \text {, } \\
0.72 \text { to } 3.28 \text { ) }\end{array}$ & $\begin{array}{l}\text { Mainly } \\
\text { negative }\end{array}$ & $1,2,3$ \\
\hline
\end{tabular}

$\mathrm{RR}=$ relative risk; $\mathrm{HR}=$ hazard ratio; NS=non-significant.

*Some studies investigated both survival and recurrence and therefore appear in table 2 also.

†In addition to psychosocial variables.

$\ddagger+=$ mainly significant associations found; $0=$ no significant associations; + --=mixed positive and negative findings.

$\S 1=$ patients recruited near to diagnosis; $2=$ adjustment for at least one confounder; $3=$ =early assessment of coping style.

medium, or low) and longer survival (hazard ratio 0.6 , 95\% confidence interval 0.4 to 0.9$).{ }^{1}$ Another large good quality study examined the impact of emotional suppression on outcome but found no significant associations with either overall or event-free survival.

\section{Publication bias}

We could not carry out standard methods of assessing publication bias such as funnel plots because there was great heterogeneity among the studies and there were only a small number of studies in each category of coping style. Studies that reported "positive" findings were smaller than those that reported non-significant findings (mean sample size $89 v 198, \mathrm{P}=0.02$, two tailed), which is indicative of publication bias. 
Table 2 Prospective studies of recurrence of cancer and coping style* (posted as supplied by authors)

\begin{tabular}{|c|c|c|c|c|c|c|c|c|}
\hline & $\begin{array}{l}\text { Sample size (\% } \\
\text { women); mean age } \\
\text { at entry (years) }\end{array}$ & $\begin{array}{l}\text { Coping style(s) } \\
\text { (scale used) }\end{array}$ & $\begin{array}{c}\text { Site of cancer and } \\
\text { stage }\end{array}$ & $\begin{array}{l}\text { Length of } \\
\text { follow up }\end{array}$ & $\begin{array}{c}\text { Adjustment for } \\
\text { confounders/ } \\
\text { examination of } \\
\text { baseline variablest }\end{array}$ & Results & $\begin{array}{c}\text { Summary } \\
\text { of } \\
\text { results } \ddagger\end{array}$ & $\begin{array}{l}\text { Summary of key } \\
\text { methodological } \\
\text { aspects of study§ }\end{array}$ \\
\hline $\begin{array}{l}\text { Brown }(2000)^{8} \\
\text { Australia }\end{array}$ & $426(39 \%) ; 53$ years & $\begin{array}{l}\text { Active, distractive and } \\
\text { avoidant coping; } \\
\text { adjustment to cancer } \\
\text { (stigma/isolation, } \\
\text { minimisation, anger) }\end{array}$ & $\begin{array}{l}\text { Early stage } \\
\text { melanoma }\end{array}$ & 2 years & $\begin{array}{l}\text { Clinical, demographic, } \\
\text { and psychological } \\
\text { factors }\end{array}$ & $\begin{array}{c}\text { Avoidant coping scores } \\
\text { and recurrence: } \\
\mathrm{HR}=1.02,95 \% \mathrm{Cl} 1.00 \text { to } \\
1.04, \mathrm{P}=0.03 . \text { Other } \\
\text { coping styles not stated }\end{array}$ & + & $1,2,3$ \\
\hline $\begin{array}{l}\text { Cassileth } \\
\quad(1985, \\
1988)^{2122} \\
\text { USA }\end{array}$ & $\begin{array}{c}\text { Group I: } \\
\text { unresectable } \\
\text { cancers; } \mathrm{n}=204 \\
(37 \%) ; 60 \text { years } \\
\text { Group II: stage I/II } \\
\text { melanoma, stage II } \\
\text { breast cancer; } \\
\mathrm{n}=155(80 \%) ; 52 \\
\text { years }\end{array}$ & $\begin{array}{l}\text { Hopelessness/ } \\
\text { helplessness, }{ }^{38} \text { amount } \\
\text { of adjustment required } \\
\text { to cope with diagnosis }\end{array}$ & $\begin{array}{c}\text { Group I: } \\
\text { unresectable } \\
\text { pancreatic, gastric, } \\
\text { non-small cell lung } \\
\text { cancer, colorectal } \\
\text { cancer, or grade } 3 \\
\text { or } 4 \text { glioma } \\
\text { Group II: melanoma } \\
\text { or breast cancer }\end{array}$ & $\begin{array}{l}\text { 1985: group I: } \\
\text { median } \\
\text { survival } 7 \\
\text { months); } \\
\text { group II: time } \\
\text { to recurrence } \\
12 \text { months. } \\
\text { 1988: } 3-8 \\
\text { years }\end{array}$ & $\begin{array}{l}\text { 1985: unadjusted. } \\
\text { 1988: diagnosis, } \\
\text { disease extent, } \\
\text { performance status, } \\
\text { marital status, age } \\
\text { group }\end{array}$ & $\begin{array}{l}\text { 1985: recurrence, no } \\
\text { unadjusted association of } \\
\text { psychosocial categories } \\
\text { with recurrence (Mantel } \\
\text { Cox statistic=4.4, } \\
\text { P>0.10). } 1988 \text { : time to } \\
\text { recurrence (group II): } \\
\text { "intermediate" level of } \\
\text { hopelessness: Cox } \\
\text { RR }=0.52,0.28 \text { to } 0.96 \text {, } \\
\mathrm{P}=0.03 \text { (adjusted for } \\
\text { psychosocial variables } \\
\text { only) }\end{array}$ & $\begin{array}{l}- \\
+\end{array}$ & $\begin{array}{c}1,3(1985) \\
1,2,3(1988)\end{array}$ \\
\hline $\begin{array}{l}\text { Dean and } \\
\text { Surtees } \\
(1989)^{15} \\
\text { UK }\end{array}$ & $\begin{array}{c}125(100 \%) ; 49 \\
\text { years }\end{array}$ & $\begin{array}{c}\text { Stoic acceptance, } \\
\text { fighting spirit, denial, } \\
\text { hopelessness/ } \\
\text { helplessness, } \\
\text { anxious/depressed }^{51}\end{array}$ & $\begin{array}{c}\text { Primary breast } \\
\text { cancer }\end{array}$ & $6-8$ years & $\begin{array}{l}\text { Age, social class, } \\
\text { marital status, } \\
\text { menopausal status, } \\
\text { employment, tumour } \\
\text { size, node status }\end{array}$ & $\begin{array}{l}\text { Patients who employed } \\
\text { hopelessness/ } \\
\text { helplessness and stoic } \\
\text { acceptance were more } \\
\text { likely to be } \\
\text { recurrence-free at follow } \\
\text { up; use of denial at } 3 \\
\text { months associated with } \\
\text { being recurrence free; no } \\
\text { other data. }\end{array}$ & + & $1,2,3$ \\
\hline $\begin{array}{l}\text { De Boer } \\
(1998)^{49} \\
\text { Netherlands }\end{array}$ & $\begin{array}{c}133 \text { (16\%); } 63 \text { years } \\
\text { (median) }\end{array}$ & $\begin{array}{l}\text { Uncertainty regarding } \\
\text { how to cope with } \\
\text { illness, or emotions }\end{array}$ & $\begin{array}{l}\text { Head, neck cancer. } \\
\text { Patients received } \\
\text { radiotherapy, } \\
\text { laryngectomy, or } \\
\text { other surgery. Stage } \\
\text { I: } 48 \% \text {, II: } 13 \% \text {, III: } \\
23 \% \text {, IV: } 21 \%\end{array}$ & 6 years & $\begin{array}{l}\text { Previous radiotherapy, } \\
\mathrm{T} \text { and } \mathrm{N} \text { classification, } \\
\text { age, education, marital } \\
\text { status, smoking, } \\
\text { drinking }\end{array}$ & $\begin{array}{c}\text { Higher uncertainty } \\
\text { regarding coping with } \\
\text { practical aspects of } \\
\text { illness (adjusted RR for } \\
\text { uncertainty score=1.03, } \\
0.96 \text { to } 1.11 \text { ); uncertainty } \\
\text { regarding coping with } \\
\text { emotions }(0.98,0.90 \text { to } \\
1.07)\end{array}$ & + & 2,3 \\
\hline $\begin{array}{l}\text { Greer }(1979)^{2} \\
\text { Pettingale } \\
(1984),{ }^{3} \\
\text { Pettingale } \\
(1985),{ }^{4} \\
\text { Greer } \\
(1990)^{5} \text { UK }\end{array}$ & $\begin{array}{c}69(100 \%) ;<70 \\
\text { years }\end{array}$ & $\begin{array}{l}\text { Denial, fighting spirit, } \\
\text { stoic acceptance, } \\
\text { helplessness/ } \\
\text { hopelessness }\end{array}$ & $\begin{array}{l}\text { Breast cancer with } \\
\text { no metastases, } \\
\text { treated by } \\
\text { mastectomy; } 25 \text { also } \\
\text { received } \\
\text { radiotherapy }\end{array}$ & $\begin{array}{c}5,10 \text {, and } 15 \\
\text { years }\end{array}$ & $\begin{array}{l}5 \text { years: none. } 10 \\
\text { years: age, } \\
\text { menopausal status, } \\
\text { stage, operation, } \\
\text { radiotherapy, histology. } \\
15 \text { years: age, } \\
\text { menopausal status, } \\
\text { stage, operation, } \\
\text { radiotherapy, histology }\end{array}$ & $\begin{array}{c}5 \text { years: recurrence or } \\
\text { death. Use of denial } \\
\mathrm{RR}=1.57,0.94 \text { to } 2.62 \text {, } \\
\text { fighting spirit } 1.88,1.19 \\
\text { to } 2.96 \text {, and either } \\
\text { coping strategy } v \\
\text { acceptance or } \\
\text { helplessness/ } \\
\text { hopelessness } 3.1,1.3 \text { to } \\
7.4 .10,15 \text { years: use of } \\
\text { denial or fighting spirit } \\
\text { predicted recurrence }\end{array}$ & + & $\begin{array}{c}1,3 \text { (at } 5 \text { years) } \\
1,2,3 \text { (at } 10 \text { years) } \\
1,2,3 \text { (at } 10 \text { years) }\end{array}$ \\
\hline $\begin{array}{l}\text { Hislop }(1987)^{41} \\
\text { Canada }\end{array}$ & $\begin{array}{l}133(100 \%) ;<55 \\
\text { years at diagnosis }\end{array}$ & $\begin{array}{l}\text { Coping by change, by } \\
\text { control, by stress }\end{array}$ & $\begin{array}{l}\text { Primary ductal } \\
\text { breast cancer }\end{array}$ & 4 years & $\begin{array}{c}\text { Age, stage, } \\
\text { pathological nodal } \\
\text { status, histological } \\
\text { grade, ER status }\end{array}$ & $\begin{array}{l}\text { No effect of coping } \\
\text { variables (high } v \text { low use } \\
\text { of coping style) on } \\
\text { recurrence. Coping by } \\
\text { change Cox adjusted } \\
\mathrm{RR}=0.6 \text {, no } \mathrm{Cl}, \mathrm{P}=0.12 \text {; } \\
\text { coping by control } 0.95 \text {, } \\
\mathrm{P}=0.9 \text {; coping by stress } \\
1.1, \mathrm{P}=0.8\end{array}$ & - & $1,2,3$ \\
\hline
\end{tabular}


Table 2 Prospective studies of recurrence of cancer and coping style* (posted as supplied by authors) contd

\begin{tabular}{|c|c|c|c|c|c|c|c|c|}
\hline & $\begin{array}{l}\text { Sample size (\% } \\
\text { women); mean age } \\
\text { at entry (years) }\end{array}$ & $\begin{array}{l}\text { Coping style(s) } \\
\text { (scale used) }\end{array}$ & $\begin{array}{c}\text { Site of cancer and } \\
\text { stage }\end{array}$ & $\begin{array}{l}\text { Length of } \\
\text { follow up }\end{array}$ & $\begin{array}{c}\text { Adjustment for } \\
\text { confounders/ } \\
\text { examination of } \\
\text { baseline variablest }\end{array}$ & Results & $\begin{array}{c}\text { Summary } \\
\text { of } \\
\text { resultsł }\end{array}$ & $\begin{array}{c}\text { Summary of key } \\
\text { methodological } \\
\text { aspects of study§ }\end{array}$ \\
\hline $\begin{array}{l}\text { Jensen } \\
\quad(1987)^{26} \\
\quad \text { USA }\end{array}$ & $\begin{array}{l}\text { Group I: women } \\
\text { with recurrence or } \\
\text { metastasis ( } \mathrm{n}=27 \text {; } \\
50 \text { years); group II: } \\
\text { women with } \\
\text { minimum } 2 \text { years in } \\
\text { remission ( } \mathrm{n}=25 ; 49 \\
\text { years); group III: } \\
\text { non-cancer controls } \\
\text { ( } \mathrm{n}=34 \text {, age not } \\
\text { given) }\end{array}$ & $\begin{array}{l}\text { Defensiveness, } \\
\text { helplessness/ } \\
\text { hopelessness, negative } \\
\text { affect, chronic stress, } \\
\text { and daydreaming } \\
\text { (Millon behavioural } \\
\text { health inventory, } \\
\mathrm{MBH}^{26} \text { ) }\end{array}$ & Breast cancer & $\begin{array}{l}\text { Average follow } \\
\text { up } 624 \text { days }\end{array}$ & $\begin{array}{c}\text { Groups similar in } \\
\text { stage, age, weight, age } \\
\text { at menarche, and first } \\
\text { pregnancy, parity, SES, } \\
\text { family history, disease } \\
\text { course, positive nodes. } \\
\text { Adjusted for age, } \\
\text { stage, cancer duration, } \\
\text { packed cell volume, } \\
\text { alkaline phosphatase }\end{array}$ & $\begin{array}{c}\text { Defensiveness (presence } \\
v \text { absence) } F=4.4 \\
P=0.04 ; \\
\text { helplessness/ } \\
\text { hopelessness score } \\
F=9.6, P=0.0006 ; \\
\text { negative affect score } \\
F=2.2, P=0.004 ; \text { chronic } \\
\text { stress } F=5.7, P=0.02 ; \\
\text { and daydreaming score } \\
F=10.4, P=0.003 \text {, were } \\
\text { independently associated } \\
\text { with clinical and vital } \\
\text { status (composite } \\
\text { variable based on final } \\
\text { disease status) }\end{array}$ & + & 1,2 \\
\hline $\begin{array}{l}\text { Rogentine } \\
(1979)^{33} \\
\text { USA }\end{array}$ & $\begin{array}{c}\text { Study 1: } 67(25 \%) ; \\
16-67 \text { years }\end{array}$ & $\begin{array}{l}\text { Adjustment needed to } \\
\text { cope with cancer } \\
\text { ("denial") })^{59}\end{array}$ & $\begin{array}{c}\text { Malignant } \\
\text { melanoma. All } \\
\text { patients disease free } \\
\text { at point of testing }\end{array}$ & $\begin{array}{c}\text { Recurrence at } \\
1 \text { year }\end{array}$ & $\begin{array}{c}\text { Stage, positive/ } \\
\text { enlarged nodes, } \\
\text { histology, Clark level, } \\
\text { location of primary, } \\
\text { age, sex, time from } \\
\text { symptoms to diagnosis }\end{array}$ & $\begin{array}{c}\text { Amount of adjustment } \\
\text { required lower in patients } \\
\text { with relapse (mean } \\
\text { score }=53 \vee 80, P<0.001 \text { ). } \\
\text { Adjustment score did not } \\
\text { correlate with number of } \\
\text { positive nodes }\end{array}$ & + & 2,3 \\
\hline $\begin{array}{l}\text { Watson } \\
(1999)^{7} \\
\text { UK }\end{array}$ & $\begin{array}{c}578(100 \%) ; 55 \\
\text { years }\end{array}$ & $\begin{array}{c}\text { Fighting spirit, } \\
\text { helplessness/ } \\
\text { hopelessness, anxious } \\
\text { preoccupation, } \\
\text { fatalism, avoidance, }{ }^{48} \\
\text { emotional suppression }\end{array}$ & $\begin{array}{c}\text { Stage I and II breast } \\
\text { cancer }\end{array}$ & 5 years & $\begin{array}{c}\text { Histopathological } \\
\text { grade, positive lymph } \\
\text { nodes, tumour size, } \\
\text { operation, } \\
\text { chemotherapy } v \\
\text { endocrine therapy, ER } \\
\text { status }\end{array}$ & $\begin{array}{l}\text { No effect of predominant } \\
\text { coping style or emotional } \\
\text { suppression on } \\
\text { event-free survival. Only } \\
\text { helplessness/ } \\
\text { hopelessness significant } \\
\text { (high } v \text { low scorers) } \\
\text { (RR=1.55, } 1.07 \text { to } 2.25) \\
\text { but not when it was } \\
\text { predominant coping } \\
\text { response }(1.15,0.60 \text { to } \\
2.22)\end{array}$ & $+1-$ & $1,2,3$ \\
\hline
\end{tabular}

$\mathrm{RR}=$ relative risk; $\mathrm{HR}=$ hazard ratio; $\mathrm{OR}=0 \mathrm{dds}$ ratio.

*Some studies investigated both recurrence and survival and therefore appear in table 1 also.

†In addition to psychosocial variables.

$\ddagger+=$ mainly significant associations found; $0=$ no significant associations; $+/==$ mixed positive and negative findings.

$\S 1=$ clearly defined group of patients recruited near to diagnosis, $2=$ adjustment for at least one confounder, $3=$ early assessment of coping style

\section{Discussion}

It is commonly believed that a person's mental attitude in response to a diagnosis of cancer affects his or her chances of survival, and the psychological coping factors that are most well known in this respect are fighting spirit and helplessness/hopelessness. ${ }^{42}$ We found little convincing evidence that either of these factors play a clinically important part in survival from or recurrence of cancer; the significant findings that do exist are confined to a few small studies. Good evidence is also lacking to support the view that "acceptance," "fatalism," or "denial" have an important influence on outcome.

Our review has several possible limitations. Firstly, the validity assessment focused on only three methodological criteria and other criteria are known to be important, such as the adequacy of baseline information. ${ }^{43}$ However, when we piloted the validity assessment checklist these criteria did not seem to differentiate adequately between the studies. We could have adopted a more stringent set of criteria, but this would be unlikely to alter the (already negative) conclusions of the review.
The review may also be subject to publication bias because the studies reporting "positive" findings tended to be smaller. We tried to identify unpublished studies, including theses and conference papers, but small studies with negative findings are less likely to be published in any form and thus may be more difficult to locate. ${ }^{44}$ Among the studies that we did identify, relatively few had adequately adjusted for important predictors of disease-free and overall survival, such as age and histological grade, ${ }^{45}$ and this is a possible explanation for some of the positive findings.

Overall we found little evidence that coping styles play an important part in survival from cancer. This is an important finding because there is often pressure on patients with cancer to engage in "positive thinking," and this may add to their psychological burden. ${ }^{46}{ }^{47}$ It has been suggested that clinicians need to detect coping styles such as helplessness or hopelessness and treat them vigorously. ${ }^{7}$ Our findings show that such interventions may be inappropriate, at least when they are used with the aim of increasing survival or reducing the risk of recurrence. 


\section{Conclusion}

Good evidence in this subject is still scarce as there have been few large methodologically sound studies. Although the relation is biologically plausible, there is at present little scientific basis for the popular lay and clinical belief that psychological coping styles have an important influence on overall or event-free survival in patients with cancer.
We are grateful to those who supplied additional data, Herman Faller, Allan House, and Sue Lockwood who commented on earlier versions of the paper, and Susan Kennedy for help with redrafting.

We carried out a supplementary search in June 2002 to update the review while it was undergoing peer review: Medline 117 additional hits; PsycLit 88 additional hits; Assia 23 additional hits; Embase 113 additional hits; Cancerlit 115 additional hits; Dissertation Abstracts 88 additional hits; Healthstar no longer existed but is now part of NLM gateway and this was searched instead, 220 additional hits from Oct 2001-June 2002; CINAHL

Table 3 Details of studies of survival and recurrence

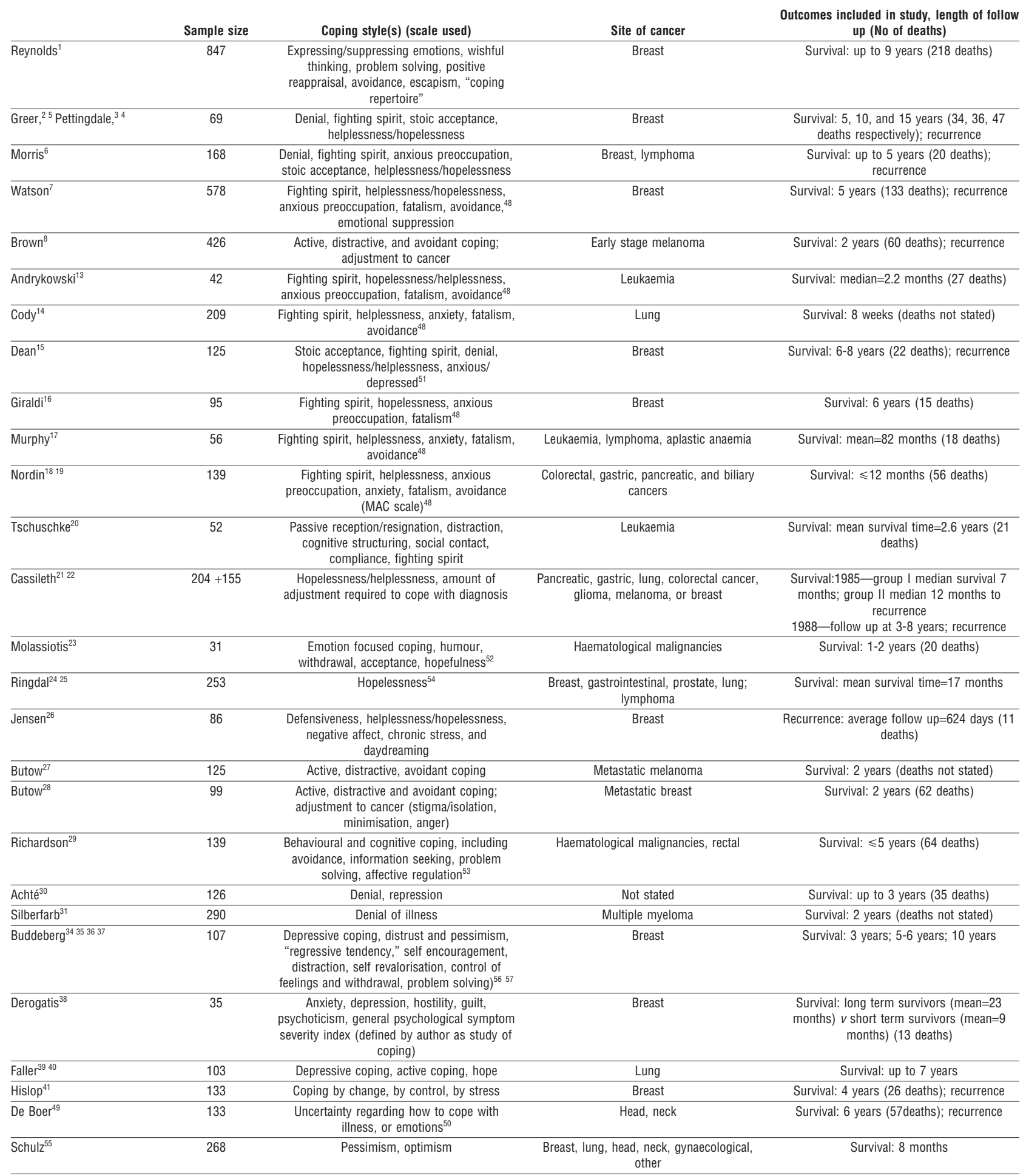




\section{What is already known on this topic}

Survival from cancer is commonly thought to be influenced by a person's psychological coping style

Some studies have shown that a coping style involving fighting spirit rather than helplessness/hopelessness is associated with survival and recurrence, though the evidence is inconsistent

\section{What this study adds}

This systematic review suggests that there is no consistent association between psychological coping and outcome of cancer

Publication bias and methodological flaws in some of the primary studies may explain some of the previous positive findings

There is no good evidence to support the development of psychological interventions to promote particular types of coping in an attempt to prolong survival

60 additional hits from Aug 2001 to June 2002. None of these abstracts was relevant to the review and none met the inclusion criteria.

Contributors: MP initiated the review and carried out the statistical analyses. MP, RB, and $\mathrm{DH}$ all contributed to the study protocol, screened abstracts, extracted data, and assessed the included studies. All authors contributed to interpreting the evidence and to writing the final paper. MP will act as guarantor.

Funding: MP is funded by the Chief Scientist Office of the Scottish Executive Department of Health and is a member of the ESRC-funded Evidence Network.

Competing interests: None declared.

1 Reynolds P, Hurley S, Torres M, Jackson J, Boyd P, Chen V. Use of coping strategies and breast cancer survival: results from the Black/White cancer survival study. Am J Epidemiol 2000;152:940-9.

2 Greer S, Morris T, Pettingale K. Psychological response to breast cancer: effect on outcome. Lancet 1979;ii:785-7.

3 Pettingale K. Coping and cancer prognosis. J Psychosom Res 1984;28:363-4.

4 Pettingale K, Morris T, Greer S, Haybittle J. Mental attitudes to cancer: an additional prognostic factor. Lancet 1985;i:750.

5 Greer S, Morris T, Pettingale K, Haybittle J. Psychological response to breast cancer and 15-year outcome. Lancet 1990:335:49-50.

6 Morris T, Pettingale K, Haybittle J. Psychological response to cancer diagnosis and disease outcome in patients with breast cancer and lymphoma. Psychooncology 1992;1:105-14.

7 Watson M, Haviland J, Greer S, Davidson J, Bliss J. Influence of psychological response on survival in breast cancer: a population-based cohort study. Lancet 1999;354: 1331-6.

8 Brown J, Butow P, Culjak G, Dunn S. Psychosocial predictors of outcome: time to relapse and survival in patients with early stage melanoma. $\mathrm{BrJ}$ Cancer 2000;83:1448-53.

9 Gerits P. Life events, coping and breast cancer: state of the art. Biomed Pharmacother 2000;54:229-33.

10 Angell M. Disease as a reflection of the psyche. $N$ Engl J Med 1985;312:1570-2.

11 Stroup D, Berlin J, Morton S, Olkin I, Williamson G, Rennie D, et al. Meta-analysis of observational studies in epidemiology: a proposal for reporting. JAMA 2000;283:2008-12.

12 NHS Centre for Reviews and Dissemination. Undertaking systematic reviews of research on effectiveness: CRD guidelines for those carrying out or commissioning reviews. York: University of York, 1996.

13 Andrykowski M, Brady M, Henslee-Downee P. Psychosocial factors predictive of survival after allogenic bone marrow transplantation for leukemia. Psychosom Med 1994;56:432-9.

14 Cody M, Nichols S, Brennan C, Armes J, Wilson P, Slevin M. Psychosocial factors and lung cancer prognosis. Psychooncology 1994;3:141.

15 Dean C, Surtees P. Do psychological factors predict survival in breast cancer? J Psychosom Res 1989;33:561-9.

16 Giraldi T, Rodani M, Cartei G, Grassi L. Psychosocial factors and breas cancer: a 6-year Italian follow-up study. Psychother Psychosom 1997;66:229 36.

17 Murphy K, Jenkins P, Whittaker J. Psychosocial morbidity and survival in adult bone marrow transplant recipients - a follow-up study. Bone Marrow Transplant 1996;18:199-201.
18 Nordin K, Glimelius B. Psychological reactions in newly diagnosed gastrointestinal cancer patients. Acta Oncol 1997;36:803-10.

19 Nordin K, Glimelius B. Reactions to gastrointestinal cancer-variation in mental adjustment and emotional well-being over time in patients with different prognoses. Psychooncology 1998;7:413-23.

20 Tschuschke V, Hertenstein B, Arnold R, Bunjes D, Denzinger R, Kaechele $\mathrm{H}$. Associations between coping and survival time of adult leukemia patients receiving allogenic bone marrow transplantation. Results of a prospective study. J Psychosom Res 2001;50:277-85.

21 Cassileth B, Walsh W, Lusk E. Psychosocial correlates of cancer survival: a subsequent report 3 to 8 years after cancer diagnosis. J Clin Oncol $1988: 6: 1753-9$.

22 Cassileth B, Lusk E, Miller D, Brown L, Miller C. Psychosocial correlates of survival in advanced malignant disease? $N$ Engl $J$ Med $1985 ; 312: 1551-5$

23 Molassiotis A, Van Den Akker O, Milligan D, Goldman J. Symptom distress, coping style and biological variables as predictors of survival after bone marrow transplantation. J Psychosom Res 1997;42:275-85.

24 Ringdal G, Gotestam K, Kaasa S, Kvinnsland S, Ringdal K. Prognostic factors and survival in a heterogeneous sample of cancer patients. $\mathrm{Br} J$ Cancer 1996;73:1594-9.

25 Ringdal G. Correlates of hopelessness in cancer patients. J Psychosoc Oncol 1995;13:47-66.

26 Jensen M. Psychobiological factors predicting the course of breast cancer. J Pers 1987;55:317-42.

27 Butow P, Coates A, Dunn S. Psychosocial predictors of survival in metastatic melanoma. J Clin Oncol 1999;17:2256-63.

28 Butow P, Coates A, Dunn S. Psychosocial predictors of survival: metastatic breast cancer. Ann Oncol 2000;11:469-74.

29 Richardson J, Zarnegar Z, Bisno B, Levine A. Psychosocial status at initiation of cancer treatment and survival. JPsychosom Res 1990;34:189-201.

30 Achté K, Vuhkonen ML, Achté A. Psychological factors and prognosis in cancer. Psych Fenn 1979:19-24.

31 Silberfarb P, Anderson K, Rundle A, Holland J, Cooper M, McIntyre O. Mood and clinical status in patients with multiple myeloma. J Clin Oncol 1991;9:2219-24.

32 Epping-Jordan J, Compas B, Howell D. Predictors of cancer progression in young adult men and women: avoidance, intrusive thoughts, and psychological symptoms. Health Psychol 1994;13:539-47.

33 Rogentine G, van Kammen D, Fox B, Docherty J, Rosenblatt J, Boyd S, et al. Psychological factors in the prognosis of malignant melanoma: a prospective study. Psychosom Med 1979;41:647-55.

34 Buddeberg C, Riehl Emde A, Landont R, Steiner R, Sieber M, Richter D. The significance of psychosocial factors for the course of breast cancerresults of a prospective follow-up study. Schweiz Arch Neurol Psychiatr 1990;141:429-55.

35 Buddeberg C, Wolf C, Sieber M, Riehl Emde A, Bergant A, Steiner R, et al. Coping strategies and course of disease of breast cancer patients. Results of a 3-year longitudinal study. Psychother Psychosom 1991:55:151-7.

36 Buddeberg C, Sieber M, Wolf C, Landolt-Ritter C, Richter D, Steiner R. Are coping strategies related to disease outcome in early breast cancer? J Psychosom Res 1996;40:255-64.

37 Buddeberg C, Buddeberg-Fischer B, Schnyder U. Coping strategies and 10-year outcome in early breast cancer. I Psychosom Res 1997;43:625-6.

38 Derogatis L, Abeloff M, Melisaratos N. Psychological coping mechanism and survival time in metastatic breast cancer. JAMA 1979;242:1504-8.

39 Faller H, Bulzebruck H, Drings P, Lang H. Coping, distress, and survival among patients with lung cancer. Arch Gen Psychiatry 1999;56:756-62.

40 Faller H, Bulzebruck H, Schilling S, Drings P, Lang H. Beeinflussen psychologische Faktoren die Uberlebenszeit bei Krebskranken? II: Ergebnisse einer empirischen Untersuchung mit Bronchialkarzinomkranken. [Do psychological factors modify survival of cancer patients? II Results of an empirical study with bronchial carcinoma patients]. Psychother Psychosom Med Psychol 1997;47:206-18

41 Hislop T, Waxler N, Coldman A, Elwood J, Khan L. The prognostic significance of psychosocial factors in women with breast cancer. J Chro Dis 1987:40:729-35.

42 Edelman S, Craig A, Kidman A. Can psychotherapy increase the survival time of cancer patients? J Psychosom Res 2000;49:149-56.

$43 \mathrm{Kahn}$ H, Sempos C. Statistical methods in epidemiology. New York: Oxford University Press, 1989

44 Gilbody S, Song F. Publication bias and the integrity of psychiatry research. Psychol Med 2000;30:253-8.

45 Sainsbury JRC, Anderson TJ, Morgan DAL. Breast cancer. BMJ 2000;321:745-50

46 Wilkinson S, Kitzinger C. Thinking differently about thinking positive: discursive approach to cancer patients' talk. Soc Sci Med 2000;50:797-811.

47 De Raeve L. Positive thinking and moral oppression in cancer care. Eur Cancer Care 1997;6:249-56.

48 Watson M, Greer S, Young J, Inayat Q, Burgess C, Robertson B. Development of a questionnaire measure of adjustment to cancer: the MAC scale. Psychol Med 1988;18:203-9.

49 De Boer M, Van den Borne B, Pruyn J, Ryckman R, Volovics L, Knegt P, et al. Psychosocial and physical correlates of survival and recurrence in patients with head and neck carcinoma: results of a 6 -year longitudinal study. Cancer 1998;83:2567-79.

50 De Boer M, Pruyn J, van den Borne B, Knegt P, Ryckman R, Verwoerd C. Rehabilitation outcomes of long-term survivors treated for head and neck cancer. Head Neck 1995;17:503-15.

51 Morris T, Greer H, White P. Psychological and social adjustment to mastectomy. Cancer 1977;40:2381-7.

52 Jalowiec A, Murphy S, Powers M. Psychometric assessment of the Jalowiec coping scale. Nurs Res 1984;33:157-61. 
53 Moos R, Cronkite P, Billings A, Finney J. Health and daily living form manual. Palo Alto, CA: Social Ecology Laboratory, Stanford University Medical Center, 1983.

54 Beck A, Weissman A, Lester D, Trexler L. The measurement of pessimism: the hopelessness scale. J Consult Clin Psychol 1974;42:861-5.

55 Schulz R, Bookwala J, Knapp J, Scheier M, Williamson G. Pessimism, age, and cancer mortality. Psychol Aging 1996;11:304-9.

56 Sieber M, Buddeberg C, Wolf C. Reliabilitat und Validitat des Zurcher Fragebogens zur Krankheitsverarbeitung (ZKV-R) [Reliability and validity of the Zurich questionnaire of coping with illness]. Schweiz Archiv Neurol Psychiatr 1991;142:553-67.
57 Muthny F. Freiburger Fragebogen zur Krankheitsverarbeitung. Weinheim, Germany: Beltz, 1989.

58 Horowitz M, Wilner N, Alvarez W. Impact of event scale: a measure of subjective stress. Psychosom Med 1979:41:209-18.

59 Rahe R. The pathway between subjects' recent life changes and their near-future illness reports: representative results and methodological issues. In: Dohrenwend B, B. Dohrenwend B, eds. Stressful life events: their nature and effects. New York: John Wiley, 1974.

(Accepted 19 July 2002) 